\title{
Correlation between serological and immunofluorescence results in the investigation of brucellosis in veterinary surgeons
}

\author{
R. J. HENDERSON, D. M. HILL, A. A. VICKERS, J. M. EDWARDS, AND \\ H. TILLETT \\ From the Royal Infirmary, and Public Health Laboratory, Worcester and the Central Public Health \\ Laboratory, Colindale, London
}

SYNOPSIS Four serological tests and three immunofluorescence tests for IgG, $\operatorname{IgM}$, and $\operatorname{IgA}$ were compared for value in the investigation of brucellosis in veterinary surgeons. No one serological test stood out over the others, and the immunofluorescence tests did not appear to have advantages over the serological tests. If a laboratory is limited in time and resources then the saline agglutination or the complement fixation test would be reasonably satisfactory. The 2-mercaptoethanol test and the antihuman globulin (Coombs' test) have no advantages over the other two and could be dropped. Immunofluorescence tests are not recommended for routine testing of brucellosis sera. The results and these recommendations apply to the 'vet' sera tested; it is reasonable to suppose that what applies to 'vet' sera will also apply to sera of those who work with or are in repeated contact with cattle and who will have had previous experience of brucella antigen, that is, dairy farmers, herdsmen, or slaughter house employees.

In a detailed clinical, radiological, haematological, and serological study of 46 veterinary surgeons the serological results and estimates of serum IgM, IgA, and IgG by immunofluorescence were related to the clinical findings and the results published (Henderson et al, 1975). This communication deals with the correlation found between the serological and immunofluorescence tests carried out in that investigation.

\section{Methods}

The serological tests were the saline agglutination test (SAT); the same test but with 0.05 м 2-mercaptoethanol in the tubes in place of saline (MET); the antihuman globulin test (AHG); and the complement fixation test (CFT). For $\operatorname{IgM}, \operatorname{IgA}$, and IgG content the method used was that of Edwards et al (1970).

Briefly, this was to put brucella antigen on a slide, dry, add the serum to be tested, allow the two to react, dry, then add either anti-IgM, anti-IgA or anti-IgG monospecific fluorescent conjugate $^{1}$ at

${ }^{1}$ Wellcome Research Laboratories

${ }^{2}$ Obtained from the Department of Experimental Pathology of Birmingham University

Received for publication 16 July 1975. optimum working dilution and incubate. Fluorescence was then read with an appropriate fluorescence microscopy apparatus. To check class specificity of the conjugates and test sensitivity 15 sera were fractionated on a sucrose density gradient and the fractions were tested in the indirect immunofluorescence test. Each conjugate stained the antigen only when a fraction with the corresponding immunoglobulin was in the serum layer. Only once was a trace of IgM apparent where it had not been revealed by the immunofluorescence test on whole serum.

As IgG and monomeric $\operatorname{IgA}$ are present in the same fractions a further test of the specificity of the anti-IgA conjugate was made. Parallel tests were set up using two sera with high $\operatorname{IgA}$ and the antiIgA conjugate, or the same conjugate after two absorptions with small amounts of pure $\operatorname{IgA}{ }^{2} \cdot \operatorname{IgA}$ was detected in the two sera at a dilution of 1 in $\mathbf{4 0}$ by the unabsorbed conjugate. After one absorption it was detected easily at a 1 in 10 dilution and after a second absorption only traces were seen at 1 in 10 . Tests for $\operatorname{IgA}$ were carried out because it was thought that IgA might play some role in the skin reactions and hypersensitivity demonstrated by some vets. When IgA is produced it appears in both serum and secretions. Anti-brucella serum $\operatorname{IgA}$ is 
certainly produced, but usually larger amounts of anti-brucella IgG are formed than of $\mathrm{IgA}$. Serum IgA may play some part in the SAT.

\section{Results}

All the vets but two gave serological reactions to one or other or all of the tests; some also gave positive tests for immunofluorescent antibodies (table 1). The two who were negative had not worked with cattle for several years. Correlation between the test results was studied using the coefficient ' $r$ ' $(r=0$ if there is none and $r=1$ if there is perfect correlation) as in table II. The best correlation of the four tests used was between AHG and CFT $(r=0.88)$. When the four tests were compared with the immunofluorescence results the best result was between AHG and IgG $(r=0.75)$.

SALINE AGgLUTINATION TEST (SAT)

This is the test most commonly used in the investigation of brucellosis and the one with which most laboratory workers are familiar. The agglutination seen in SAT is probably due, principally, to the presence of IgG, but there is usually some IgM and also possibly IgA. The nine highest titres (table I) related to vets in their early 20 s or 30 s, five of whom were symptomless.

ANTIHUMAN GLOBULIN (COOMBS') TEST (AHG) The AHG results are worth examining. Vets often ask for this test to be done when their sera are being

\begin{tabular}{|c|c|c|c|c|c|c|c|}
\hline Case & $S A T$ & $M E T$ & $A H G$ & $C F T$ & $\operatorname{Ig} M$ & $\operatorname{Ig} A$ & $\operatorname{Ig} G$ \\
\hline 1 & 160 & $<10$ & 1280 & 40 & $<10$ & $\operatorname{Tr} 10$ & 20 \\
\hline 2 & 160 & 160 & 2560 & 160 & $<10$ & $\operatorname{Tr} 10$ & 20 \\
\hline 3 & 80 & 80 & 640 & 10 & $<10$ & $<10$ & 10 \\
\hline 4 & 80 & 40 & $>5120$ & 80 & $<10$ & $\operatorname{Tr} 10$ & 10 \\
\hline 5 & 320 & 80 & 2560 & 40 & $<10$ & $\operatorname{Tr} 10$ & 10 \\
\hline 6 & 40 & 40 & 640 & 10 & $<10$ & $<10$ & 10 \\
\hline 7 & 320 & 320 & 2560 & 80 & $<10$ & $<10$ & 10 \\
\hline 8 & 20 & 20 & 2560 & 160 & $<10$ & 40 & 40 \\
\hline 9 & 160 & 80 & $>10240$ & 640 & $<10$ & $<10$ & 20 \\
\hline 10 & 80 & 20 & 320 & 10 & $<10$ & $<10$ & $<10$ \\
\hline 11 & 160 & 160 & $>10240$ & 640 & $<10$ & 10 & 80 \\
\hline 12 & 20 & $<10$ & 80 & 5 & $<10$ & $<10$ & $<10$ \\
\hline 13 & 40 & $<10$ & 80 & 5 & $<10$ & $<10$ & $<10$ \\
\hline 14 & 80 & 20 & 2560 & 80 & $<10$ & $<10$ & 40 \\
\hline 15 & 160 & 80 & 1280 & 40 & $<10$ & $<10$ & 40 \\
\hline 16 & $<10$ & $<10$ & 1280 & 80 & $\operatorname{Tr} 10$ & $\operatorname{Tr} 10$ & 40 \\
\hline 17 & $<10$ & $<10$ & $>10$ & $<10$ & $<10$ & $<10$ & $<10$ \\
\hline 18 & $<10$ & $<10$ & 2560 & 160 & $<10$ & $<10$ & 40 \\
\hline 19 & $<10$ & $<10$ & 160 & 20 & $<10$ & $<10$ & 10 \\
\hline 20 & 160 & 80 & 640 & 40 & $<10$ & $\operatorname{Tr} 10$ & 40 \\
\hline 21 & 320 & 320 & 5120 & 160 & $\operatorname{Tr} 10$ & 20 & 160 \\
\hline 22 & 160 & 80 & $>2650$ & 320 & $<10$ & 40 & 80 \\
\hline 23 & 640 & 640 & 2560 & 320 & $<10$ & 10 & 640 \\
\hline 24 & $<10$ & $<10$ & $<10$ & $<10$ & $<10$ & $<10$ & $<10$ \\
\hline 25 & 80 & 90 & 2560 & 80 & $<10$ & $\operatorname{Tr} 10$ & 20 \\
\hline 26 & 160 & 20 & 1280 & 160 & $<10$ & $<10$ & 20 \\
\hline 27 & 160 & 40 & 640 & 20 & $<10$ & $<10$ & 20 \\
\hline 28 & 160 & $<10$ & 160 & 10 & $<10$ & $<10$ & $<10$ \\
\hline 29 & 1280 & 320 & 2560 & 320 & 80 & 20 & 40 \\
\hline 30 & 640 & 40 & 2560 & 320 & $<10$ & 80 & 80 \\
\hline 31 & $<10$ & $<10$ & 80 & $<10$ & $<10$ & $<10$ & $\operatorname{Tr} 10$ \\
\hline 32 & 640 & $<10$ & 640 & 160 & 20 & 10 & 10 \\
\hline 33 & $<10$ & 20 & 1280 & 160 & $>10$ & 10 & 40 \\
\hline 34 & 320 & 160 & 2560 & 320 & $>10$ & 10 & 40 \\
\hline 35 & 160 & 40 & 640 & 40 & $>10$ & 10 & 10 \\
\hline 36 & 80 & 80 & 2560 & 160 & $>10$ & 10 & 40 \\
\hline 37 & 40 & 20 & 80 & $<10$ & $>10$ & $<10$ & $\operatorname{Tr} 10$ \\
\hline 38 & 80 & 40 & 640 & 10 & $>10$ & $<10$ & $<10$ \\
\hline 39 & 640 & 160 & 1280 & 40 & 10 & 40 & 40 \\
\hline 40 & 1280 & 1280 & $>5120$ & 160 & $<10$ & 40 & 80 \\
\hline 41 & 640 & 160 & 1280 & 20 & 20 & 40 & 80 \\
\hline 42 & 640 & 640 & 2560 & 320 & $\operatorname{Tr} 10$ & 10 & 10 \\
\hline 43 & 640 & 640 & 5120 & 80 & 10 & 10 & 160 \\
\hline 44 & $<10$ & $<10$ & 640 & 10 & $<10$ & 10 & 20 \\
\hline 45 & 20 & 20 & 1280 & 40 & $<10$ & 10 & 40 \\
\hline 46 & 20 & 20 & 160 & $<10$ & $<10$ & $<10$ & $\operatorname{Tr} 10$ \\
\hline
\end{tabular}

Table I Results of serological tests in 46 vets 


\begin{tabular}{|c|c|c|c|c|c|c|c|c|c|c|}
\hline \multirow{2}{*}{$\begin{array}{l}\text { Serum Antibody Levels } \\
\text { by CF Test }\end{array}$} & \multicolumn{9}{|c|}{ Serum Antibody Levels by AHG Test } & \multirow[t]{2}{*}{ Total } \\
\hline & - & 80 & 160 & 320 & 640 & 1280 & 2560 & 5120 & $>5120$ & \\
\hline- & 2 & 2 & 1 & - & - & - & - & & - & 5 \\
\hline 5 & - & 2 & - & - & - & - & - & - & - & 2 \\
\hline 10 & - & - & 1 & 1 & 4 & - & - & & - & 6 \\
\hline 20 & - & - & 1 & - & 1 & 1 & - & - & - & 3 \\
\hline 40 & - & - & - & - & 2 & 4 & 1 & - & - & 7 \\
\hline 80 & - & - & - & - & - & $i$ & 3 & 1 & 1 & 6 \\
\hline 160 & - & - & - & - & 1 & 2 & 4 & 1 & 1 & 9 \\
\hline 320 & - & - & - & - & - & - & 5 & 1 & - & 6 \\
\hline 640 & - & - & - & - & - & - & - & - & 2 & 2 \\
\hline Total & 2 & 4 & 3 & 1 & 8 & 8 & 13 & 3 & 4 & 46 \\
\hline
\end{tabular}

Table II Correlation between serological and immunofluorescence results in the investigation of brucellosis in veterinary surgeons

If the coefficient ' $r$ ' is taken as a measure of correlation ( $r=0$ if there is none and $r=1$ if there is perfect correlation) then the result for AHG and CFT as in table $I I$ is $r=0.88$.

This was the best correlation between tests. The value of ' $r$ ' for other comparisons is AHG and SAT, $r=0.57 ; A H G$ and MET, $r=0.66$; AHG and IgG, $r=0.75 ;$ CFT and $\mathrm{IgG}, \mathrm{r}=0.74 ; \mathrm{MET}$ and $\mathrm{IgG}, \mathrm{r}=0.53$; SAT and CFT, $\mathrm{r}=0.54 ; \mathrm{SAT}$ and $\mathrm{MET}, \mathrm{r}=0.77$;

SAT and $I g G, r=0.45 ; C F T$ and $M E T, r=0.55$.

There were too many sera without IgA and IgM to calculate correlation coefficients.

examined and attach great importance to the results. It is difficult to see the rationale for this. The test detects antibody that has been formed because of the presence of foreign antigen, and it is useful in revealing the presence of a portion of antibody present in the serum which does not easily form a lattice with the antigen but will do so when the antibody molecules which are combined with antigen are linked together by the antiglobulin agent. It is not possible to say why this particular type of antibody should be present in vets' sera unless it be as a result of repeated exposure to infection but it is not established that it is not found in all brucella sera. In fact it might be found in all antibacterial sera if it were looked for. Of the 46 vets examined, 44 had titres ranging from 80 to 10240 by the AHG test.

\section{2-MERCAPTOETHANOL TEST (MET)}

This test has been used to demonstrate indirectly the presence of serum IgM, because 2-mercaptoethanol breaks the disulphide links of the IgM pentamer, thus interfering with its highly efficient agglutinating capacity while not affecting IgG molecules. The SAT result represents principally agglutination by IgG and IgM; any reduction therefore in result between SAT and MET would indicate the amount of $\operatorname{IgM}$ in the serum. Thus it was thought that this test would help to differentiate between recent exposure to Brucella abortus and longstanding or chronic disease because IgM is usually formed early in response to infection and IgG later. There were inconsistencies in the use of the test since in the 23 instances (table I) where MET was less than SAT, fluorescence microscopy detected IgM in only four of the 23 .

\section{COMPLEMENT FIXATION TEST (CFT)}

Forty-one vets had complement fixing antibody titres ranging from 5 to 640 ; the remaining sera were negative. Comparisons between CFT and AHG gave the highest correlation found $(r=0.88)$ but $r$ values with SAT and MET were significantly lower.

\section{IMMUNOFLUORESCENCE}

IgM was detected in eight sera, IgA in 27 , and IgG in 39. Correlation was best between IgG and CFT $(0.74)$ and between IgG and AHG $(0.75)$. Calculations of ' $r$ ' were made only for IgG. There were too many sera without IgM and IgA to make calculations of ' $r$ ' values for these immunoglobulins possible. IgM was detected in only eight sera. In three the value of IgM was trace only at a 1/10 serum dilution but in one young vet IgM was detectable at a serum dilution of $1 / 80$. In this instance there was a reduction in MET compared with SAT which fitted with the IgM demonstrated. He also had a high AHG and CFT but he was symptomless. There was a decrease in proportions of vets with IgM in the older age groups; thus IgM was found in $5 / 13$ vets under 30 years, in $2 / 16$ between 30 to 40 , and in $1 / 17$ over 50.

\section{Discussion}

The primary object of this investigation into brucellosis and veterinary surgeons was to relate tests to clinical findings, but it was thought that the analysis of laboratory results might be of value in helping laboratory workers to decide which tests to use because testing sera for brucellosis antibodies 
has become time-consuming and laborious owing to the number of tests that have been introduced and because blood culture is seldom successful. The tests showed a varying correlation, highest between AHG and CFT, and AHG and IgG. This could be expected as IgG is the immunoglobulin produced in greatest quantity and persists longest, but nothing emerged from the comparisons to suggest that any of the four tests was particularly better as a diagnostic weapon than any other or that immunofluorescence tests could replace the usual serological tests. It appeared that one or at the most two serological tests were all that was necessary for the serological investigation of brucellosis.

Immunofluorescence tests were investigated in the hope that they might bear a better relation to clinical findings than the serological tests but this did not prove to be so and they cannot be recommended for use in routine laboratory work. As for serological tests the value of the MET was doubtful to say the least, and it is for consideration whether it and the AHG, or Coombs' test as it is known, could not be dropped. The Coombs' test is very time-consuming and although haematological laboratories usually have an automatic apparatus for this particular test, bacteriological laboratories might not be so well equipped. Without such equipment the centrifuging and washing of the deposit does take up a lot of laboratory time, especially if more than one serum is to be tested. The information the MET test gave was of no value and that from the
AHG bore no particularly better relation to the clinical findings than did the SAT and CFT.

If a laboratory worker has the time and wishes to confirm a saline agglutination result then the CFT would probably be the easiest test to fit into the routine work as most laboratories already do $\mathrm{CF}$ testing, but the lesson from the work described in this and its companion paper is that the taking of a detailed history and a physical examination is probably the most important factor in the investigation of brucellosis in vets, and if a single serological test is required the SAT would do as well as any. It must be repeated, however, that the view expressed here applies only to those who work with infected cattle and are subjected to repeated infection, that is, vets, dairy farmers, and slaughter house workers. If any member of the general public, without previous experience of the antigen, is unfortunate enough to become infected with brucellae then serological examination would obviously be of the greatest importance.

We thank Mr J. Benjamin for help with the serological tests.

\section{References}

Edwards, J. M. B., Tannahill, A., and Bradstreet, P. (1970). Comparison of the indirect fluorescent antibody test with agglutination, complement-fixation, and Coombs' tests for Brucella antibody. J. Clin. Path., 23, 161-165.

Henderson, R. J., Hill, D. M., Vickers, A. A., Edwards, J. M. B., and Tillett, H. E. (1975). Brucellosis and veterinary surgeons. Brit. Med. J., 2, 656-659. 